\title{
Les Jeunes-Turcs dans la littérature grecque : entre légende et réalité
}

The Young Turks in Greek litterature: between legend and reality

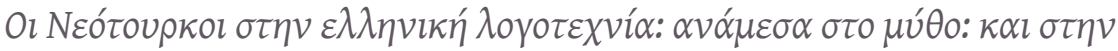

$\pi \rho \alpha \gamma \mu \alpha \tau \imath \kappa o ́ \tau \eta \tau \alpha$

\section{Georges Kostakiotis}

\section{OpenEdition}

\section{Journals}

Édition électronique

URL : https://journals.openedition.org/ceb/1042

DOI : $10.4000 /$ ceb. 1042

ISSN : 2261-4184

Éditeur

INALCO

Édition imprimée

Date de publication : 9 janvier 2012

ISSN : 0290-7402

Référence électronique

Georges Kostakiotis, "Les Jeunes-Turcs dans la littérature grecque : entre légende et réalité », Cahiers balkaniques [En ligne], 40 | 2012, mis en ligne le 27 mai 2012, consulté le 06 juillet 2021. URL : http:// journals.openedition.org/ceb/1042 ; DOI : https://doi.org/10.4000/ceb.1042

Ce document a été généré automatiquement le 6 juillet 2021

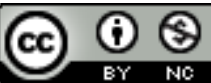

Cahiers balkaniques est mis à disposition selon les termes de la Licence Creative Commons Attribution - Pas d'Utilisation Commerciale 4.0 International. 


\title{
Les Jeunes-Turcs dans la littérature grecque : entre légende et réalité
}

\author{
The Young Turks in Greek litterature: between legend and reality

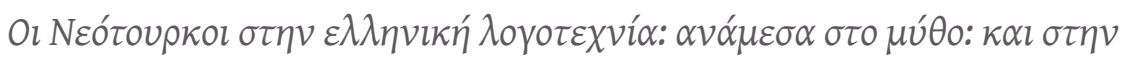 \\ $\pi \rho \alpha \gamma \mu \alpha \tau \imath \kappa o ́ \tau \eta \tau \alpha$
}

Georges Kostakiotis

1 Le mouvement des «Jeunes-Turcs» qui bouleversa le monde des Balkans et l'espace ottoman en général ne pouvait pas laisser indifférente la littérature grecque. Malgré la place limitée qu'elle semble lui consacrer, elle lui accorde, nous semble-t-il, une signification importante et déterminante au sein du récit.

2 Dans cet article, nous essaierons de comprendre comment, à travers leurs héros, les écrivains grecs intègrent ce mouvement dans la littérature et donc dans la mémoire collective grecque.

3 Pour cette étude, nous avons choisi de porter notre regard sur trois œuvres différentes qui marquent, chacune avec son style particulier et son propre point de vue, son époque. Il s'agit de la nouvelle de Stratis Myrivilis, Vassilis l'Arvanite, publiée ${ }^{3}$ en 1943, du roman de Christos Samouïlidis, Karamanites, Les Derniers Grecs de Cappadoce, publié en 1965 et de celui de Réa Stathopoulou, Le Roi des Chiffres, paru en 2006.

4 La question des Jeunes-Turcs n'occupe la scène principale dans aucune de ces trois œuvres néanmoins elle joue un rôle déterminant dans l'évolution de l'intrigue. Chez Myrivilis et Samouïlidis, la révolution est placée exactement au milieu du récit et marque le changement, le passage ou bien le basculement d'un monde dans un autre. Réa Stathopoulou, elle, raconte le mouvement des Jeunes-Turcs plus tôt, au début de son roman et témoigne du climat politique et du contexte historique et social dans lequel tout un siècle va se dérouler. 


\section{Myrivilis}

5 Myrivilis rend compte de la révolution des Jeunes-Turcs à partir de deux grands événements : d'une part, les célébrations collectives afin d'oublier désormais la haine du passé et de se donner la main, et, d'autre part, l'amnistie générale accordée à tous les prisonniers suivie de leur libération immédiate. Notons qu'il s'agit d'événements qui concernent la petite société fermée du village qui reste assez loin de la politique et des centres de décision.

Dans sa nouvelle, Myrivilis décrit la personnalité hors du commun de Vassilis l'Arvanite et évoque avec précision la société de son enfance, au début du siècle, dans le monde rural des villages de l'île de Lesbos. L'héroïsme et le caractère sans égal du protagoniste évoquent l'héroïsme de la mythologie et de l'histoire grecques. Déçu par la situation du monde de son époque, Myrivilis se replie sur lui-même et tente un retour vers les valeurs et les idéaux d'autres époques. Le mépris de Vassilis pour la société fermée et ses institutions dépassées, son mépris pour l'occupant turc, font allusion au dédain que le peuple manifeste envers la dictature de Métaxas, envers les années noires, la Deuxième Guerre mondiale et l'occupation allemande.

7 L'absence d'une idéologie politique claire et ouvertement dénonciatrice permettra aux régimes politiques de récupérer l'œuvre de Myrivilis, notamment cette nouvelle, ce qui va minimiser le refus de Vassilis de se soumettre à l'autorité et mettra davantage l'accent sur son héroïsme mythique. Très justement, Menis Koumantareas ${ }^{4}$, prenant part à une polémique autour de l'œuvre de Myrivilis, fait la part des choses et explique l'importance du travail de l'écrivain qui prend donc ses distances avec la politique sans pour autant ignorer ni ses propres faiblesses ni le contexte politico historique ${ }^{5}$.

8 Le mouvement des Jeunes-Turcs joue un rôle majeur dans l'œuvre de Myrivilis bien qu'il n'occupe qu'à peine deux ou trois pages. L'évocation de ce mouvement est placée exactement au milieu du récit et marque le début d'un nouveau départ. Il ne concerne pas seulement le changement d'un régime, la disparition d'un tyran ou la réactivation d'une constitution. Il va bien plus loin, abolissant les frontières, l'idée de patrie ou la notion de nation. Bien évidemment, l'écrivain n'ignore rien de la réalité du monde qu'il met en scène et il n'a pas l'intention ou le but de bousculer les traditions ancestrales et leurs codes religieusement respectés; il ne tente pas d'embellir cet univers malgré la nostalgie évidente qu'il éprouve. Après la mise en place du cadre de son histoire, la présentation des personnages dans les premiers chapitres, il va nous parler des relations entre les Grecs et les Turcs dans le village: sans détour et d'une phrase simple, directe et courte il nous dit, - il nous rappelle - qu'entre les deux communautés les contacts sont loin d'être considérés comme paisibles:

"Nous avions pas mal de Turcs dans le village en ce temps-là, et la haine était profonde entre les deux peuples.

Nous cohabitions paisiblement, mais uniquement de loin, dans la stricte mesure où nous avions besoin les uns des autres. À eux le pouvoir, à nous les terres, l'astuce et les piastres. Séparément nos cafés, séparément nos réjouissances, séparément nos fêtes " ${ }^{6}$.

9 L'absence du verbe et la répétition trois fois de suite du mot " $\chi \omega ́ p l \alpha$ », « séparément ", rendent avec justesse une réalité que, souvent, les sociologues et les historiens ainsi que ceux qui cultivent les souvenirs nostalgiques d'un passé idéalisé ont tendance à occulter, voire à minimiser. L'auteur ne cherche pas davantage à justifier ce comportement; Myrivilis, dans le rôle très complexe de narrateur, d'acteur et 
d'observateur, prend ses distances avec un regard devenant parfois cynique comme dans la scène qui décrit l'enterrement d'un Turc qui ne serait, pour lui, qu'un chien crevép. Il parle ouvertement et dénonce clairement le fanatisme. Le cliché du Grec gentil, victime du turc méchant tombe : le Grec comme le Turc montrent désormais leurs vrais sentiments qui sont loin d'être nobles.

10 Myrivilis raconte sans états d'âme une réalité, sèche, dure et choquante, pour faire ressentir le changement qui va suivre et marquer le contraste avec l'arrivée des JeunesTurcs au pouvoir. C'est pourquoi, trois chapitres plus loin, alors que le lecteur était désormais plongé dans le microcosme du village et suivait les aventures de la vie quotidienne avec tout de même deux tueries, la révolution de Thessalonique vient bouleverser le monde et changer le cours de l'histoire ainsi que les codes d'une organisation ancestrale : « Nous devions dorénavant oublier nos vieilles querelles, nous serrer la main $»^{8}$.

11 Le mouvement de Thessalonique arrive jusqu'à ce village de l'île de Lesbos pour réconcilier les habitants, pour leur faire oublier les rivalités dans un monde qui semble désormais dépassé. Si les Jeunes-Turcs, avec leur révolution de juin 1908, demandent des changements politiques, des droits égaux et la liberté d'expression, les villageois, plus terre à terre, loin des préoccupations "constitutionnelles", ressentent les résultats immédiats dans leurs relations avec leurs voisins qui, jusqu'au chapitre précédent, n'étaient que des ennemis. Pour eux, la révolution proprement dite, n'est que l'ordre officiel qui arrive au village pour demander

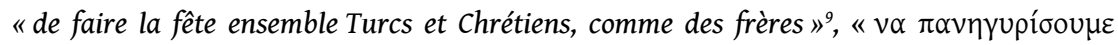

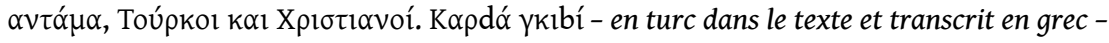

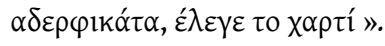

12 C'est pour cette raison que l'annonce de la constitution qui arrive brusquement surprend aussi bien les villageois que le lecteur averti ${ }^{10}$.

«Et puis un jour, sans qu'on s'y attende, arriva la Constitution turque! Ce fut une éruption volcanique qui bouleversa tout un monde. Incroyable et extraordinaire qu'en quelques jours plus rien ne reste du pouvoir et de la grandeur immenses du sultan, du Padichah. Et de surcroît de ce Hamid dont même les Turcs ne prononçaient le nom qu'à voix basse en regardant autour d'eux si personne ne les entendait. Les Jeunes-Turcs le déposèrent, prirent le pouvoir et enjoignirent à tous les peuples de l'Empire de célébrer l'événement historique que nous vivions".

13 L'écrivain utilise alors toute une série de symboles pour montrer le bouleversement: nous suivons de ce fait les célébrations, la messe des chrétiens et la prière des musulmans en commun avec la participation du curé et du hodja. On note également le geste significatif du hodja qui noue ensemble les drapeaux grec et turc devant une foule prise au dépourvu, qui applaudit et qui a des frissons, quand les deux chefs religieux tombent dans les bras l'un de l'autre pour s'embrasser. Myrivilis insiste: «c'est une chose inouïe pour ce monde ! ${ }^{11}$. Les élèves grecs et turcs, avec leurs professeurs, se retrouvent sur la place du village, ils sont endimanchés, portent des rameaux, mais ressentent un grand trouble: ils n'avaient l'habitude de se croiser que pour jouer à la guerre et se lancer des pierres.

Ces deux communautés qui se côtoient et qui, pourtant se méprisent et se haïssent, se retrouvent d'un instant à l'autre unifiées. Les Turcs s'étonnent, mais ils obéissent comme toujours, note Myrivilis avec une certaine ironie, tandis que les Grecs considèrent ce changement comme un pas vers leur libération. C'est pour cette raison que la décoration des maisons, des cafés, des écoles, des églises avec des lauriers et des 
drapeaux grecs, semble quelque peu excessive ; le narrateur se demande d'ailleurs, d'où ils ont pu sortir autant de drapeaux aussi grands ${ }^{12}$ et si bien cachés.

Vient ensuite la libération des prisonniers ${ }^{13}$, il s'agit là, très certainement, d'un acte politique hautement symbolique pour montrer l'abolition du pouvoir du sultan. Mais pour le village, cet acte prend une toute autre dimension très concrète puisque c'est le héros du village, le jeune et brave Vassilis, qui revient librement après des années d'exil suite à l'assassinat d'un Turc. Il n'a plus peur de se faire arrêter. Au contraire, il est accueilli chaleureusement, il fait la fête et expose avec fierté ses décorations: l'une grecque, décernée par le prince grec pour avoir participé au combat pour la libération de la Macédoine (où il a tué des comitadjis et des soldats turcs comme des moustiques) et une autre, turque cette fois ci, pour avoir combattu aux côtés des Jeunes-Turcs à Constantinople même, contre le sultan. Myrivilis fait allusion à la deuxième phase du mouvement quand l'armée de Macédoine est parvenue jusqu'à la capitale, pour mettre fin à la contre-révolution qui s'était organisée en avril 1909 et qui a mené à la destitution du sultan ${ }^{14}$.

Dans cette nouvelle ce n'est pas tant l'événement historique qui nous intéresse, mais c'est surtout le très surprenant comportement du protagoniste. Cette fois-ci, ce n'est pas la foule anonyme, mais les chefs de la communauté qui ont la parole : «c'est-à-dire mon fils que tu as laissé tomber le combat national et que tu es parti. Tu as laissé tomber notre Macédoine, » lui dit le directeur de l'école, et « toi Vassilis, qu'est-ce que tu avais à partager avec le sultan?» s'étonne le notable. Les gens ont du mal à comprendre le comportement du jeune homme : laisser la patrie pour lutter aux côtés des Turcs, c'est une chose incompréhensible pour les Grecs. Myrivilis, à travers cette personnalité hors du commun, pose le problème moral de la position difficile et délicate des chrétiens dans l'Empire ottoman au tournant du siècle, au moment de la création des États nationaux. Devant le dilemme entre Empire ottoman et patrie libre, le choix ne semblait pas difficile à faire. Cependant l'écrivain va beaucoup plus loin avec la réponse quelque peu surprenante de Vassilis. Le héros est le porteur des valeurs de la société et en même temps c'est lui qui va trahir ${ }^{15}$ ces valeurs :

"Vassilis haussa les épaules. Le regard fixé au loin, au-dessus des arbres, par-delà des maisons, il dit: 'Avec les antartes - les résistants - en Macédoine, ou avec le Comité à Constantinople, c'était pareil, guerre d'un côté, guerre aussi de l'autre. Sauf qu'à Constantinople, on se battait contre un sultan. Ça avait une autre allure' $»^{16}$.

17 Avec son héros étonnamment naï, qui laisse perplexe tout un village, l'écrivain devient cynique. Avec une certaine ironie, il utilise le mouvement des Jeunes-Turcs, qu'il place à l'origine du nouvel État national et du dépassement des frontières, pour expliquer les évolutions. Cette société fermée, traditionnelle, bien loin de la capitale ou de la grande métropole de Macédoine, deviendra finalement un élément précurseur pour proposer de nouveaux idéaux et de l'espoir. Myrivilis qui a bien connu toutes les guerres qui ont marqué le début du siècle n'hésite pas, en 1943, à mépriser l'occupant par la voix d'un héros qui ne s'est jamais soumis à quelque autorité que ce soit et dont la liberté d'esprit dépasse largement les États et leurs projets nationalistes. Vassilis, l'Arvanite annonce, d'une certaine façon, l'arrivée d'Alexis Zorba de Nikos Kazantzakis qui très probablement aurait lu la nouvelle de Myrivilis et qui, dans le même esprit, en aurait poursuivi le développement ${ }^{17}$. 


\section{Samouïlidis}

18 Considérons maintenant la deuxième œuvre choisie, Karamanites, Les Derniers Grecs de Cappadoce. Si Myrivilis, depuis Lesbos, fait allusion aux combats de son protagoniste dans les rangs de l'armée des Jeunes-Turcs et d'Enver Pacha contre le sultan à Constantinople, chez Samouïlidis la révolution des Jeunes-Turcs se situe à Adana et en plein massacre des Arméniens. Il s'agit cette fois d'un roman historique au sens propre du terme, dans lequel durant quelque 450 pages nous suivons, à travers le personnage principal, l'histoire d'un village grec de Cappadoce, pendant une période qui va du dernier quart du XIXe siècle jusqu'à l'échange des populations après la signature du Traité de Lausanne.

19 Samouilidis est bien documenté, puisqu'il a travaillé pendant 15 ans aux côtés d'Octave et Melpo Merlier au centre d'Études d'Asie Mineure. Il y a enregistré et répertorié les témoignages des exilés après la Catastrophe de Smyrne. Il nous propose une œuvre historique et ethnographique dense et riche. D'une écriture simple, avec des descriptions vives et concrètes, dans une langue directe et précise qui « rend » le parler local et les influences turques, il obtient une composition complexe qui retrace les étapes de l'histoire, en donnant des éléments et des informations sur l'héritage culturel.

Dans l'œuvre de Samouilidis, la révolution des Jeunes-Turcs est aussi ressentie comme un volcan qui explose pour bouleverser le monde ; l'écrivain nous prépare à ce choc, il nous permet de suivre les changements du climat et des saisons avec des images tantôt bucoliques, tantôt dramatiques et pathétiques. Le printemps, avec les couleurs et les odeurs du mois d'avril et les fêtes de Pâques composent un tableau vivant en parfait accord avec les affaires florissantes du protagoniste ${ }^{18}$. Pourtant plus nous nous approchons de la révolution, plus le temps devient lourd et la chaleur étouffante. L'ambiance change radicalement, et la douceur estivale se transforme en supplice intenable pour les habitants de la région:

"On avait l'impression que l'été de 1908 était arrivé avant l'heure. Dès la fin mai, déjà il y avait de telles chaleurs que beaucoup d'agriculteurs souffraient d'insolation. La moisson avait commencé en s'essoufflant, comme un supplice, dure »... «Le mois de juin était passé et juillet était arrivé encore plus brûlant. Les jours, en flammes et secs, traînaient jusqu'au crépuscule essoufflés. Tout ce qu'on touchait, tout ce qu'on voyait était brûlant! les pierres, les arbres, les rues, les murs, les animaux, les hommes, les cieux, les nuages, le vent ! on avait l'impression qu'un volcan avait explosé en cachette et versé sa lave invisible sur le monde. D'un instant à l'autre, d'un moment à l'autre les gens se préparaient à entendre une explosion terrible! $»^{19}$

21 Nous pouvons nous poser alors un certain nombre de questions :

22 Est-ce pour annoncer une explosion qui va apaiser ce monde de souffrance et améliorer la vie de ces gens qui sont anéantis et incapables de réagir devant cette injustice venue du ciel?

Cette chaleur ne serait-elle pas une allusion à l'autorité du sultan qui oppresse et qui tue?

Cette situation qui ne peut plus durer, peut-être ne peut-on la changer qu'avec une explosion, autrement dit qu'avec une révolution?

L'écrivain désavoue-t-il le mouvement des Jeunes-Turcs, veut-il annoncer le mal qui arrive et qui va peser sur la communauté grecque? 
d'accomplir, au titre de légaité, le service miltaire qui, jusquà mântenant, était d'accomplir, au titre de l'égalité, le service militaire qui, jusqu'à maintenant, était réservé aux seuls Turcs. L'écrivain nous fait partager la foi de son héros pour la nouvelle politique; il y croit à tel point, qu'il décide de s'y engager pleinement. Samouilidis se donne ainsi du temps pour parler de l'organisation du "Comité de l'union et du progrès », et plonge son lecteur dans l'ambiance politique des semaines qui ont suivi les manifestations dans les rues. Fidèle à l'histoire, l'auteur nous rappelle les problèmes d'organisation, souligne le caractère secret qui dure, même après la déclaration de la prise du pouvoir par le mouvement et le rétablissement de la Constitution. Il note l'absence de ligne directrice, d'idéologie ferme et précise, et en fin de compte, il note également le manque de chef politique ou militaire pour conduire le mouvement. 
ottoman. Il met en contact son protagoniste avec un Turc, un de ses amis de Thessalonique, qui lui apprend les vrais objectifs du mouvement des Jeunes-Turcs. C'est peut-être maintenant, que l'explosion du volcan a lieu: «Dorénavant lui dit-il, confidentiellement, en Turquie, seuls vivront ceux qui se considéreront comme Turcs ». Lorsqu'il entend ces paroles, un frisson traverse notre Grec, sa déception est très vive : "Alors les Jeunes-Turcs feraient le contraire de ce qu'ils déclaraient officiellement? Alors toute cette affaire n'était qu'une tromperie?» s'étonne notre héros et il continue : «Quelle terrible déception ! $»^{25}$

À partir de ce moment, l'auteur retire son protagoniste de l'engagement politique et développe une critique sévère envers le mouvement des Jeunes-Turcs : il parle du mécontentement général. Non seulement il évoque celui des communautés grecques et arméniennes qui ont vu rapidement le grand danger qui les menaçait ${ }^{26}$, mais il évoque aussi le mécontentement de $80 \%$ des Turcs qui se montrent indifférents, voire hostiles

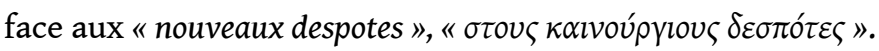

Samouïlidis devient même caustique en soulignant qu'une foule "de petits sultans",

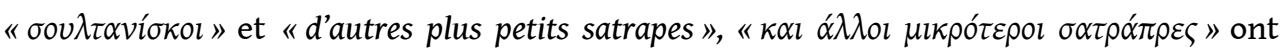
succédé au sultan et il va jusqu'à parler de membres fanatisés du Comité qui sèment la haine envers les autres communautés dans le cœur des Turcs jusqu'alors pacifiques.

L'écrivain intègre dans son récit le massacre des Arméniens ${ }^{27}$ qui a lieu le mardi de Pâques 1909 et marque le début du génocide. Il fait de saisissantes et cruelles descriptions et conclut en parlant d'une tragédie "inimaginable », " $\alpha \sigma u ́ \lambda \lambda \eta \pi \tau \eta$ ", qui a

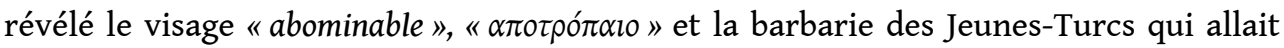
frapper aussi les Grecs $^{28}$ : "Qui pourrait garantir que le même sort n'attendait pas les Grecs? ${ }^{29}$.

Alors que Myrivilis met l'accent sur la coexistence pacifique (le mouvement de Thessalonique est ressenti plutôt comme un changement qui bouleverse le quotidien puisque Grecs et Turcs doivent "se serrer la main»), Samouïlidis écrit un texte plus politique. Le protagoniste, malgré les hésitations et les sous-entendus de l'écrivain, fait preuve de bonne foi et montre sa volonté de se laisser entraîner dans l'aventure de "l'union et du progrès ", mais il sera vite profondément déçu et découragé. Dans une langue désormais dure et crue, l'écrivain prend sa revanche face à la naïveté de son héros. Volontairement et consciemment, Samouilidis présente ces événements d'un point de vue grec, avec la ferme intention de les inscrire dans l'histoire du déracinement de l'hellénisme en Asie. C'est sans doute pour cette raison, qu'il nous offre une image plus générale en citant les journaux de l'époque : "il voyait que tous parlaient d'une seule voix, de la transformation de la vie en Turquie, du progrès et du développement économique $»^{30}$. Il est clair que le regard des uns sur la situation politique ne correspond pas à celui des autres.

\section{Stathopoulou}

Intéressons-nous maintenant au roman de Réa Stathopoulou : Le Roi des Chiffres, qui se déroule cette fois-ci dans la capitale, à Constantinople. Il s'agit plus d'une saga familiale que d'un roman historique. Mais le récit suit pourtant les événements politiques et décrit des situations qui appartiennent à l'histoire. Il est question, dans Le Roi des Chiffres, de l'histoire familiale de l'écrivain, originaire de Constantinople; l'auteur 
explique dans son introduction que cette histoire est basée sur les souvenirs de son propre père.

La langue de Réa Stathopoulou, malgré l'utilisation d'expressions idiomatiques, pour rendre le parler constantinopolitain, et malgré la présence de nombreux termes turcs, reste très moderne et actuelle. Elle nous offre une composition réaliste et crédible, basée sur des dialogues en style direct ou indirect de gens ordinaires, tels qu'on les rencontre dans la vie quotidienne.

Le narrateur se fait discret : il ne fait que rapporter les événements, sans intervenir dans le déroulement de l'action. Si Samouïlidis est l'inventeur de ses héros, Réa Stathopoulou, elle, semble observer l'action de ses personnages surgis de sa mémoire familiale. La romancière semble vouloir imiter le style de Maria Iordanidou et elle parvient à nous présenter un monde qui rappelle celui de Loxandra.

commence en 1907 pour se terminer après 1909. Par conséquent, la révolution des Jeunes-Turcs se situe presque au début du livre; elle coïncide avec la naissance du père et va jouer un rôle capital puisque les résultats de sa politique vont déterminer la vie de la famille et de toute la communauté grecque de Constantinople ${ }^{31}$. Réa Stathopoulou associe la naissance de son protagoniste au mouvement des JeunesTurcs; elle relie en réalité le destin de l'hellénisme constantinopolitain à la naissance de la Turquie moderne et met en parallèle deux mondes opposés, obligés de coexister jusqu'à l'extinction de l'un ou de l'autre.

Réa Staphopoulou, dans son roman, ne présente pas le mouvement des «Jeunes-Turcs » comme une explosion volcanique qui surprend le monde: nous sommes dans la capitale, les gens suivent l'actualité, lisent les journaux et semblent informés de l'agitation politique à Thessalonique plusieurs mois avant la fin juillet 1908. Dès le mois de février, ses héros se préparaient et à prendre position ${ }^{32}$. C'est pourquoi, devant les hommes de la communauté qui pouvaient soutenir et partager les idéaux de la révolution, l'hésitation, la suspicion et l'incertitude viennent freiner l'enthousiasme: "Il y avait aussi certains Grecs, qui voyaient avec beaucoup de réserves ce mouvement ", note l'écrivain. Parmi eux, le Patriarche œcuménique Joaquim III, qui soulignait clairement et sans détour, dans des discussions privées, qu'il «ne faut pas faire croire de telles choses et que les Turcs sont des Turcs $»^{33}$.

Ces paroles reviendront plus tard pour souligner la méfiance des Grecs envers les réformes promises ${ }^{34}$. D'ailleurs, sur un ton ironique, mais aussi plein de rancœur, le protagoniste joue avec l'adjectif « jeune », « véoৎ » qui qualifie les Jeunes-Turcs. Dans la langue grecque, cet adjectif a la double signification: «jeune» et «nouveau» et Stathopoulou, sur un ton moqueur, note que le Turc ne peut pas être nouveau, ne peut pas changer.

45 C'est dans cette perspective que l'écrivain, à l'opposé de Myrivilis qui surprend avec «la fraternité » naïvement déclarée de son héros et contrairement à Samouïlidis qui laisse son protagoniste être tenté par la révolution avant de la dénoncer, coupe court à la volonté de certains Grecs de croire aux bonnes intentions des Turcs. L'écrivain ne tient pas compte de la déclaration de la révolution à Thessalonique et des manifestations de la foule dans les rues, comme à Adana et à Lesbos. Elle se concentre principalement sur la question du service militaire, désormais obligatoire, pour tous les citoyens de la Turquie et, seulement à cette occasion, nous fait un exposé historique rapide et précis. Elle ne manque pas, pour autant, de mentionner l'effervescence 
générale dans la communauté grecque. Comme dans les deux autres œuvres que nous avons étudiées, cette effervescence pousse les gens à croire qu'ils vont jouer un rôle important ${ }^{35}$. Réa Stathopoulou considère cet enthousiasme comme un signe de faiblesse. Elle souligne amèrement que: "la promesse pour des droits égaux fait que beaucoup de Grecs sont prêts à discuter aussi de la question du service militaire dans l'armée turque ». Bien qu'elle ne manque pas d'un certain réalisme, en soulignant qu'il faut accomplir les devoirs pour pouvoir exiger des droits égaux ${ }^{36}$, cette nouvelle annonce déjà pour son protagoniste, les jours difficiles qui vont venir : " son cœur est devenu lourd comme s'il avait pressenti la menace ${ }^{37}$.

De la même façon, la question du nouveau système électoral, mis en place pour les élections au Parlement ottoman de novembre et décembre 1908, préoccupe les protagonistes. Les Grecs jugent le système trop injuste à leur égard, car ce système a pour but de diminuer le nombre de leurs représentants. Pour cette raison, d'après le Progrès, un des journaux grecs de l'époque cité dans le roman, une manifestation est organisée par les Grecs le 9 novembre. Partis de la Grand Rue du Péra, ils se dirigent vers la Sublime Porte en criant, comme les Jeunes-Turcs : «Liberté, Justice, Égalité ». C'est la cavalerie, continue le reportage, qui a arrêté le cortège sur le pont de Galata. Elle a blessé de nombreuses personnes, et l'une d'entre elles a été transférée à l'hôpital dans le coma ${ }^{38}$.

Réa Stathopoulou comme Samouïlidis avant elle nous fait un commentaire sur les journaux qui, ne subissant plus la censure, écrivaient tout ce qu'ils voulaient ${ }^{39}$. L'écrivain fait allusion à la liberté de la presse. Cette liberté a permis, en 1908-1909, à quelque 350 journaux et périodiques de voir le jour dans l'Empire, chiffre qui, à lui seul, situe la frénésie qui s'était emparée de la Turquie ${ }^{40}$. L'auteur mentionne également des grèves qui ont lieu pour la première fois à Constantinople ${ }^{41}$ et qui deviendront incontrôlables dans le courant du mois d'avril 1909, comme celle des porteurs de la douane : «Même les porteurs, se sont soulevés et demandaient leurs droits en faisant grève $~_{42}$ note l'écrivain avec une certaine ironie pour ajouter sur un ton plus dramatique que le pays était quasiment sans gouvernement : «les derniers jours, la grande cité était presque non gouvernée $»^{43}$.

48 L'auteur nous parle aussi de la contre-révolution organisée à Constantinople, le 31 mars. Réa Stathopoulou mentionne qu'une grande foule, dirigée par les imams a massacré les officiers à l'aide de la garde de la ville ${ }^{44}$. Ce soulèvement a provoqué en retour l'intervention immédiate de l'armée des Jeunes-Turcs, venue directement de

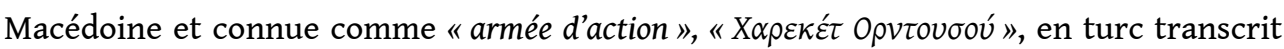
avec des caractères grecs dans le texte. La romancière nous raconte ensuite la destitution du sultan et son exil à Thessalonique.

C'est en courant, que le protagoniste arrive pour annoncer que le "gaga-burunlu $»^{45}$, littéralement celui qui a le nez crochu a été renvoyé. Malgré une méfiance et une hésitation certaines, les Grecs finissent par exprimer leur satisfaction suite à la chute $\mathrm{du}$ sultan. Ils osent désormais parler librement et oublient les espions du régime qui, jusqu'à ce jour, faisaient la loi ${ }^{46}$.

50 Néanmoins, avant de tourner la page de cette histoire, la narratrice remarque avec amertume que ses protagonistes n'imaginaient même pas que les moments difficiles qu'ils allaient vivre pousseraient certains d'entre eux à se rappeler avec nostalgie l'époque du tyran sanguinaire ${ }^{47}$. Il est évident que l'écrivain, probablement du fait de ses origines et de ses propres expériences, se montre beaucoup plus sensible aux 
questions qui concernent les réformes touchant directement la communauté grecque. Par conséquent, elle se veut encore plus critique, voire sévère et négative, que Samouilidis. Elle n'ignore rien de l'ambiance générale et obtient un équilibre parfait entre les histoires de la famille, la vie de la communauté et les grandes étapes de l'histoire turque.

51 Le mouvement des Jeunes-Turcs, qui bouleversa le monde des Balkans et l'espace ottoman en général n'a pas laissé indifférent le monde grec. Considérés d'une part comme une composante de l'Empire ottoman, à ce titre impliqués directement dans les réformes et changements politiques, et d'autre part, comme citoyens d'un pays voisin en guerre revendiquant des territoires encore occupés par les Ottomans, les Grecs ont accordé une place significative au mouvement des Jeunes-Turcs non seulement dans leur historiographie, mais aussi dans leur littérature.

\section{Conclusion}

52 Avec les trois œuvres que nous venons d'étudier, nous constatons qu'il existe un réel intérêt dans la littérature grecque pour le mouvement des Jeunes-Turcs et nous suivons les différents points de vue de chaque écrivain selon sa sensibilité, selon son époque et surtout selon ses intentions. Bien que pour les trois auteurs, le mouvement de Macédoine soit vécu comme une occasion d'améliorer la condition des populations grecques et donc, comme une étape pour accéder à la liberté, chaque écrivain l'intègre différemment dans son récit.

Pour Stratis Myrivilis qui parait être le plus conservateur des trois, le mouvement des Jeunes-Turcs est considéré comme une invitation à dépasser les frontières et les différends nationaux ; son message de fraternité se lit comme une provocation à l'égard de l'occupant.

Christos Samouïlidis quant à lui, bien que fidèle à la vérité historique, paraît plus critique ; le mouvement des Jeunes-Turcs avec les faux espoirs et la désillusion rapide $\mathrm{du}$ héros devient de façon très dramatique le moment du basculement qui marquera « le commencement de la fin » du monde grec en Asie Mineure.

Réa Stathopoulou, de son côté, se montre la plus réaliste. Jamais vraiment enchantée, elle garde ses distances: ses protagonistes suivent de près les événements et s'intéressent à la vie politique; ils essaient de comprendre leur monde et tentent de prendre en main leur destin.

\section{BIBLIOGRAPHIE}

Agathos, Thanasis, (2008), Zorbas et Vassilis l'Arvanite, (en grec) Anti, Athènes, $\mathrm{n}^{\circ}$ 911-912, pp. 35-39.

Dimadis, K. A., (2004), Dictature, Guerre et Littérature (en grec), Athènes : Estia. 
Dumont, Paul et Georgeon, François, (1989), « La mort d'un empire (1908-1923) », Histoire de l'Empire ottoman, Paris : Fayard, pp. 577-647.

Karvelis, Takis, (1996), « Stratis Myrivilis », La littérature de l'entre-deux guerres. De la Première à la Deuxième Guerre mondiale, (en grec) Athènes : Sokolis, pp. 96-136.

Koumantareas, Menis, (2010), Vassilis, l'Arvanite. Un gaillard en colère, (en grec) Nea Estia, Athènes, février, $\mathrm{n}^{\circ}$ 1830, vol. 167, pp. 287-297.

Myrivilis, Stratis, (2006), Vassilis, l'Arvanite, (en grec) Athènes : Estia, (1943).

Samouïlidis, Christos, (1999), Karamanites, Les Derniers Grecs de Cappadoce, (en grec) Athènes : Estia (1965).

Stathopoulou, Réa, (2006), Le Roi des Chiffres, (en grec), Athènes : Okeanida.

Tziovas, Dimitris, (2007), L'autre être, Identité et Société dans la Prose Néohellénique, (en grec)

Athènes : Polis.

\section{NOTES}

1. Publiée pour la première fois en 1934, ensuite en 1939 et définitivement en 1943.

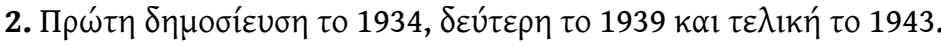

3. Publiée pour la première fois en 1934, ensuite en 1939 et définitivement en 1943.

4. Koumantareas, 2010, pp. 287-297.

5. Dimadis, 2004, pp. 172-188.

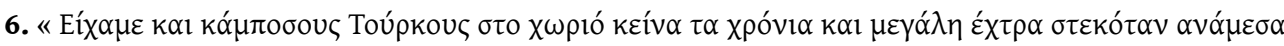

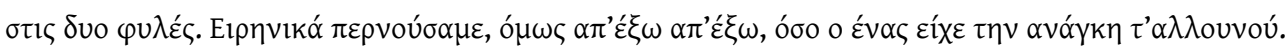

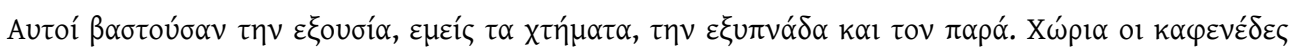

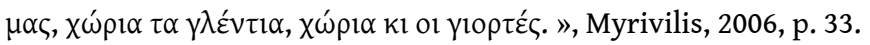

7. Myrivilis, 2006, p. 33.

8. Ibidem, p. 51.

9. Ibid., p. 51.

10. Myrivilis, 2006, p. 51.

11. Ibidem, p. 52.

12. Ibid., pp. 52, 53.

13. Ibid., p. 53.

14. Dumont-Georgeon, 1989, pp. 582-584.

15. Tziovas, 2007, p. 294.

16. Myrivilis, 2006, p. 57.

17. Myrivilis publie en 1943, Kazantzakis en 1946. Voir à ce propos, Agathos, 2008, pp. 35-39.

18. Samouilidis, 1999, p. 247.

19. Ibidem, p. 248.

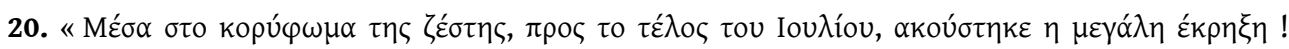

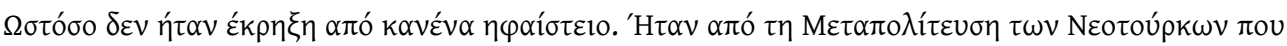

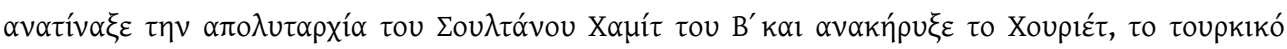
$\Sigma u ́ v \tau \alpha \gamma \mu \alpha$. », Samouïlidis, 1999, p. 249.

21. Idem.

22. Ibid., p. 250.

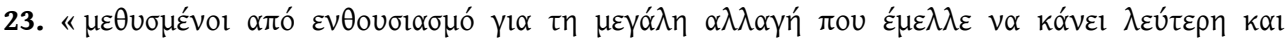

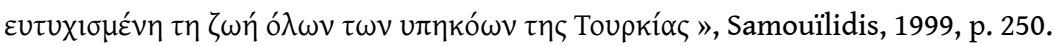

24. Ibid., p. 251. 
25. Samouilidis, 1999, p. 252.

26. Idem.

27. Dumont-Georgeon, 1989, p. 583.

28. Samouillidis, 1999, p. 258.

29. Ibidem, p. 259.

30. Samouïlidis, 1999, p. 259.

31. À rappeler que les Grecs de la capitale comme ceux des îles d'Imbros et de Ténédos ont été exemptés de l'échange des populations.

32. Stathopoulou, 2006, p. 36.

33. Stathopoulou, 2006, p. 37, 38.

34. Ibidem, p. 47.

35. Ibid., pp. 42-43.

36. Ibid., p. 42.

37. Ibid., p. 44.

38. Stathopoulou, 2006, pp. 46-47. Voir aussi Dumont-Georgeon, 1989, pp. 580, 581.

39. Stathopoulou, 2006, p. 43.

40. Dumont-Georgeon, 1989, p. 585.

41. Stathopoulou, 2006, p. 43. Voir aussi Dumont-Georgeon, 1989, p. 587.

42. Stathopoulou, 2006, p. 57.

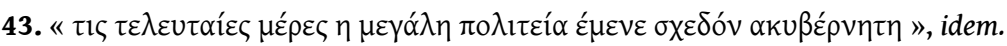

44. Stathopoulou, 2006, p. 57. Voir aussi Dumont-Georgeon, 1989, p. 583.

45. Stathopoulou, 2006, p. 59.

46. Stathopoulou, 2006, p. 59.

47. Idem.

\section{RÉSUMÉS}

Les Jeunes-Turcs dans la nouvelle de Myrivilis, Vassilis l'Arvanite, publiée ${ }^{1}$ en 1943, le roman de Samouilidis, Karamanites, publié en 1965 et celui de Réa Stathopoulou, Le Roi des Chiffres, paru en 2006, des textes écrits par des écrivains issus de milieux différents en des temps différents et qui décrivent des milieux différents. Pour les trois, le mouvement de Thessalonique inaugure une ère riche d'espoirs, Liberté, Égalité, Justice enthousiasment les populations, mais si Myrivilis reste fidèle à ce message de fraternité, Samouïlidis et Réa Stathopoulou, plus réalistes, construisant des récits qui portent sur la longue durée, sont plus critiques : nul n'imaginait qu'il en viendrait à regretter un jour le « bon vieux temps » d'Abd-ul-Hamid !

In the novel "Vasilis Arvanitis", by Myrivilis, published in 1943, as well as in the novel "Karamanites", by Samouilidis, published in 1965, and finally in "the King of Figures" of Rea Stathopoulou, published in 2006, the Young Turks are being mentioned. All three books were written by writers coming from different backgrounds, belonging to different eras, while the three writers focus on different social backgrounds. For all three of them, the movement of Thessaloniki marks the beginning of an era rich in hope, history, equality and justice, intoxicating the masses with this message. Myrivilis focuses more on the message of fraternity, while Samouilidis ans Rea Stathopoulou, stay closer to realism, while they construct their 
narration more around the long term duration, and criticism. Nobody could fathom that someday, one would come to reminisce with nostalgia the "good old days" of Abd-ul-Hamid.

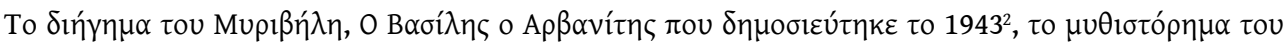

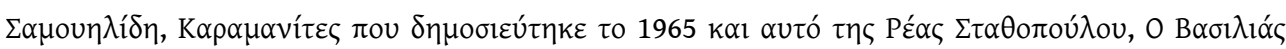

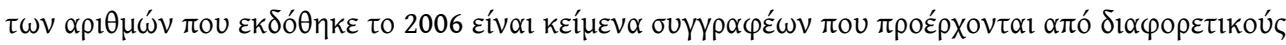

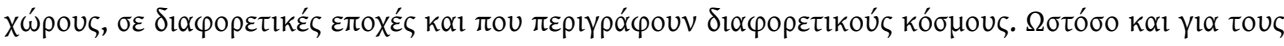

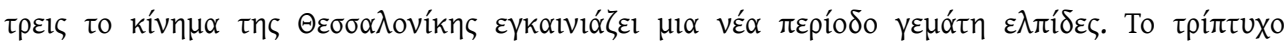

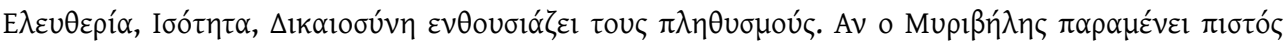

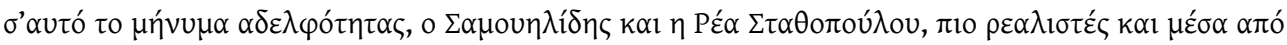

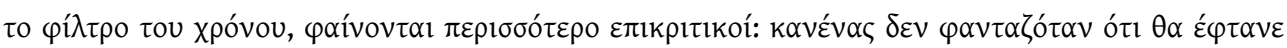

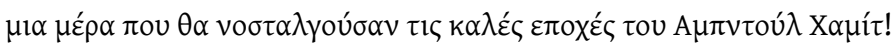

\section{INDEX}

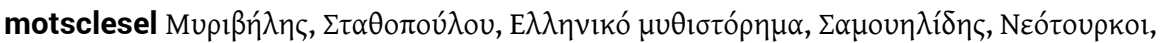

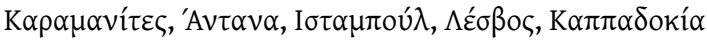

Index géographique : Adana, Istanbul, Lesbos, Cappadoce

Index chronologique : révolution jeune-turque (1908-1914)

motsclestr Adana, İstanbul, Lesbos, Capadokya, Genç Türkler, Kapadokya

motsclesmk ИСТАНБУЛ, КАПАДОКИЈА, ЛЕЗБОС, МЛАДОТУРСКАТА

Thèmes : Littérature, Histoire

Mots-clés : Circassien, Comitadji, roman grec, Hodja, Samouïlidis Christos (1927-), Karaman/

Karamanli, Myrivilis Stratis (1890-1969), Jeunes-Turcs, Samouïlidis Christos (1927-), Stathopoulou Réa (1950-)

Keywords : Myrivilis Stratis (1890-1969), Young Turks, greek novel, Istanbul, Lesbos, Cappadocia, Young Turks revolution (1908-1914), Literature, Stathopoulou Rea (1950-), Adana 